\title{
Estimation of Maximum Drift of MDOF Shear Structures Using Only One Accelerometer
}

\author{
Kangqian $\mathrm{Xu}^{1, \mathrm{a},{ }^{*}}$ and Akira Mita ${ }^{1, \mathrm{~b}}$ \\ 1. Department of System Design Engineering, Keio University, Yokohama, Japan \\ axukangqian@keio.jp, bmita@sd.keio.ac.jp
}

\begin{abstract}
Keywords: Maximum Inter-Story Drift, Shear Structure, Modal Coordinates, StateSpace Equation, Kalman Filter
\end{abstract}

\begin{abstract}
This paper presents a new method to estimate maximum drifts, relative displacements between adjacent floors, of all stories of multi-degree-of-freedom (MDOF) shear structures using only one floor's absolute acceleration time history response under the ground excitation. The absolute acceleration and relative displacement are formulated in modal coordinates and the state-space expression is derived. Then the numerical simulation for a three-story structure was conducted to verify the performance of the state-space equation. The comparison of the estimated state and input with actual values is made and shows the good agreement. In addition, the relative displacement time histories of all floors were obtained, and the errors of maximum displacements and inter-story drifts were analyzed. The robustness against environmental noise was also investigated by numerical simulations as well. The results of simulations indicate the estimation is satisfactory, and very robust against the environmental disturbance.
\end{abstract}

\section{Introduction}

The performance of civil infrastructures, such as buildings or bridges, gradually deteriorates under service loads and damage could occur when structures suffer from disasters, such as earthquakes. Here, structural health monitoring (SHM) has been extensively researched over the last few decades as a means of assessing the states of structures [1].

Maximum inter-story drift (drift angle) is an important parameter with which to evaluate the state of a structure. Inter-story drift is relative to the structural deformation capacity, and it is widely used in the design stage to judge whether a building will meet seismic design codes [2] or standards $[3,4]$. In the service stage, significant inter-story drift intuitively suggests there may be damage to structural and non-structural members [5]. For seismic isolation structures and supplementally damped buildings, the peak inter-story drift significantly affect the characteristics of bearings and dampers and consequently the dynamic properties of the structures [6-10].

Acceleration responses are easier to detect than displacements in buildings [11], and the accuracy of accelerometers is normally higher than that of displacement sensors. The displacement time history responses relative to the ground can be obtained from the integral of the relative accelerations or a Kalman filter $[12,13]$, which requires several accelerometers and at least one on the base. These accelerometers need power and data cables, the installation of which entails a significant amount of labor. Moreover, such cables could be damaged, resulting in failure of the sensors during an extreme hazard. To overcome these difficulties, wireless passive sensors $[14,15]$ based on radiofrequency identification (RFID) or other technologies have been studied. In addition, identification schemes relying on fewer sensors have studied. For example, Yoshimoto et al. [16] used only three accelerometers to detect damage to base-isolated 
buildings whose models were simplified by combining linear interpolation or cosine interpolation with the substructure approach. Considering that structures are usually still elastic or slightly non-linear during most earthquakes, we propose a novel way to estimate the maximum relative displacement of all floors in a linear multi-degree-of-freedom (MDOF) structure merely from the acceleration time history response recorded by one sensor installed on a floor.

This paper is organized as follows: Section 2 formulates the structural responses in modal coordinates. Section 3 discusses the results of numerical simulations investigating the applicability and robustness against environmental noise of the proposed approach. Section 4 summarizes this study and points out the potential of this research.

\section{Formulation of Proposed Method}

As shown in Figure 1, the equation of motion of a MDOF structure that is subjected to seismic excitation can be described as:

$$
\mathbf{M} \ddot{\mathbf{z}}(t)+\mathbf{C} \dot{\mathbf{z}}(t)+\mathbf{K z}(t)=-\mathbf{M r} \ddot{u}_{g}(t)
$$

where $\mathbf{z}(t)$ denotes the displacement relative to the ground, $(\cdot)$ means the time derivative,

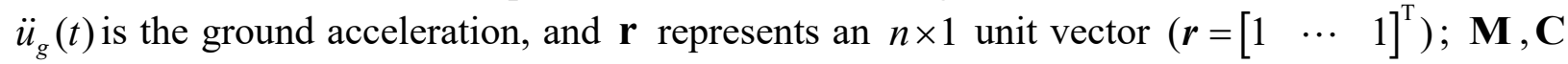
and $\mathbf{K}$ are the structural mass, damping and stiffness matrixes, respectively.

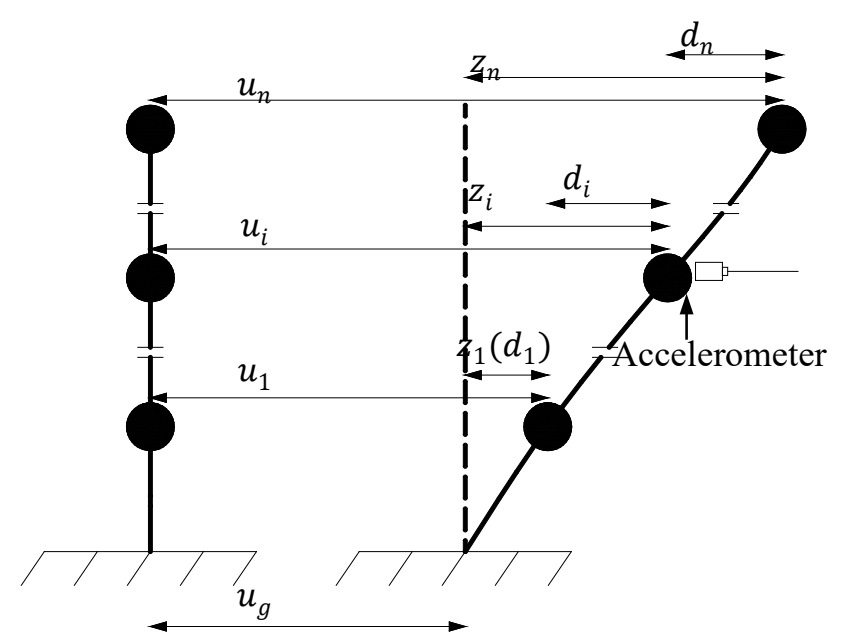

Figure 1. MDOF structure subjected to seismic excitation

By installing one accelerometer on a floor, we can obtain the floor's absolute acceleration time history response $\ddot{u}_{i}(t)$. We will focus on the estimation of the maximum relative displacement $z_{i}(t)_{\max }$ or subsequently the inter-story drift $d_{i}(t)_{\max }$ of the floor based on the absolute acceleration response.

The relative displacement of the $i$ th DOF of the MDOF structure can be written in modal coordinates:

$$
z_{i}(t)=\sum_{k=1}^{n} \phi_{i k} \eta_{k}(t)
$$


where $\Phi$ represents the mode shapes matrix of the structure, $\eta(t)$ is the modal coordinate, the subscript $i$ means the DOF whose response is observed, and the subscript $k$ denotes the participation mode.

After obtaining the natural frequencies and damping ratios of the structure, we can calculate the modal coordinates by using the Duhamel integral when the structure is excited by a seismic wave $\ddot{u}_{g}(t)$ :

$$
\eta_{k}(t)=\frac{\Gamma_{k}}{\omega_{D k}} \int_{0}^{t} \ddot{u}_{g}(\tau) \sin \omega_{D k}(t-\tau) \exp \left[-\xi_{k} \omega_{k}(t-\tau)\right] d \tau=\Gamma_{k} v_{k}(t)
$$

where $\omega$ indicates the natural frequency of the undamped system, the subscript $D$ denotes the parameter in damped system, $\xi$ is the damping ratio, $v_{k}(t)$ is defined as the modal displacement response caused by a SDOF system whose dynamic parameters are equal to the corresponding parameters in the $k$ th mode of the MDOF structure under the ground excitation $\ddot{u}_{g}(t)$, and $\Gamma$ represents the participation factor:

$$
\Gamma_{k}=\phi_{k}^{\mathrm{T}} \mathbf{M r}
$$

The equation of motion in modal coordinates with mass normalized mode shape gives:

$$
\ddot{\eta}_{k}(t)+2 \xi_{k} \omega_{k} \dot{\eta}_{k}(t)+\omega_{k}^{2} \eta_{k}(t)=-\phi_{k}^{\mathrm{T}} \mathbf{M r} \ddot{u}_{g}(t), k=1,2 \cdots n
$$

By substituting Equations (3) and its first time derivative $\dot{\eta}_{k}(t)$ into (5), the relative acceleration in modal coordinates $\ddot{\eta}_{k}(t)$ can be calculated.

As a result, the absolute acceleration of the $i$ th floor in physical coordinates can be expressed as:

$$
\ddot{u}_{i}(t)=\sum_{k=1}^{n}\left(\phi_{i k}\left(\begin{array}{c}
\left(2 \xi_{k}^{2} \omega_{k}-\frac{\omega_{k}^{2}}{\omega_{D k}}\right) \Gamma_{k} \int_{0}^{t} \ddot{u}_{g}(\tau) \sin \omega_{D k}(t-\tau) \exp \left[-\xi_{k} \omega_{k}(t-\tau)\right] d \tau \\
-2 \xi_{k} \omega_{k} \Gamma_{k} \int_{0}^{t} \ddot{u}_{g}(\tau) \cos \omega_{D k}(t-\tau) \exp \left[-\xi_{k} \omega_{k}(t-\tau)\right] d \tau
\end{array}\right)\right)
$$

If we neglect the terms including the damping ratio in the equation [17], the absolute acceleration of the measured DOF can be approximately formulated as:

$$
\ddot{u}_{i}(t)=\sum_{k=1}^{n} \omega_{k}^{2} \phi_{i k} \eta_{k}(t)=\sum_{k=1}^{n} \omega_{k}^{2} \phi_{i k} \Gamma_{k} v_{k}(t)
$$

We use the modal displacement response $v_{k}(t)$ and modal velocity response $\dot{v}_{k}(t)$ to constitute the state-space equation. Consequently, the state-space equation in continuous time can be formulated as:

$$
\begin{aligned}
\dot{\mathbf{x}}_{k} & =\mathbf{A}_{c} \mathbf{x}_{k}+\mathbf{B}_{c} \mathbf{p}_{k}+\mathbf{w}_{k}^{x} \\
\mathbf{y}_{k} & =\mathbf{H}_{c} \mathbf{x}_{k}+\mathbf{v}_{k} \\
\mathbf{z}_{k} & =\mathbf{L}_{c} \mathbf{x}_{k}
\end{aligned}
$$


where the process noise $\mathbf{w}_{k}^{x}$ and measurement noise $\mathbf{v}_{k}$ are assumed to be white, zero-mean, uncorrelated, with known covariance matrixes $\mathbf{Q}^{x}$ and $\mathbf{R}$, respectively, and the system matrixes are formed by modal parameters.

In the process equation, the input $\mathbf{p}_{k}$, that is the seismic wave $\ddot{u}_{g}(t)$, is unknown because there is no accelerometer on the structural base to record the acceleration. Similar to the reference [13], we use a additional fictitious process combined with a Gaussian random walk model to estimate the input and state simultaneously.

\section{Numerical Simulation}

Numerical simulations were conducted to verify the proposed approach of estimating the relative displacement and inter-story drift from the absolute acceleration of a story. A three-story structure is considered in this section. Figure 2 presents the model together with the structural parameters, including the damped mass and inter-story stiffness. The damping ratios in the first and second modes were assumed as $2 \%$ and $3 \%$, respectively, and the damping ratio in the third mode was decided by Rayleigh damping. The structural natural frequencies and damping ratios are listed in Table 1.

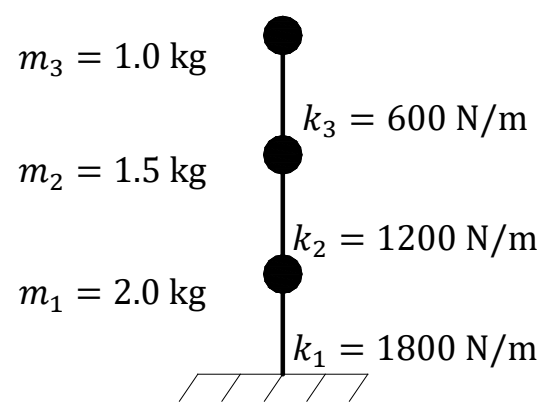

Figure 2. Simulation model of the three-DOF structure

Table 1. Modal information of the three-DOF structure

\begin{tabular}{ccc}
\hline & Frequency $(\mathrm{Hz})$ & Damping ratio (\%) \\
\hline First & 2.31 & 2.00 \\
Second & 4.94 & 3.00 \\
Third & 7.34 & 4.16 \\
\hline
\end{tabular}

The typical ground acceleration, El Centro earthquake wave (NS component), was taken as an example. The structural time history responses were analyzed by Newmark beta method. We selected the absolute acceleration of the first floor as the observation data. The measurement noise $\mathbf{R}$, process noise $\mathbf{Q}^{x}, \mathbf{Q}^{p}$ of the state and input were set to $10^{-2} \mathbf{I}, 10^{-4} \mathbf{I}$ and $10^{10} \mathbf{I}$ ( $\mathbf{I}$ is an identify matrix of appropriate dimension), respectively. The input and state including the modal displacement responses $v_{k}\left(\omega_{k}, \xi_{k}, \ddot{u}_{g}, t\right)$ and modal velocity responses $\dot{v}_{k}\left(\omega_{k}, \xi_{k}, \ddot{u}_{g}, t\right)$ were evaluated using the Kalman filter. As can be seen in Figure 3, the filter could make a highly accurate estimation of the external force, and the responses in higher modes were recovered as were the lower modal vibrations regardless of their low amplitudes. 


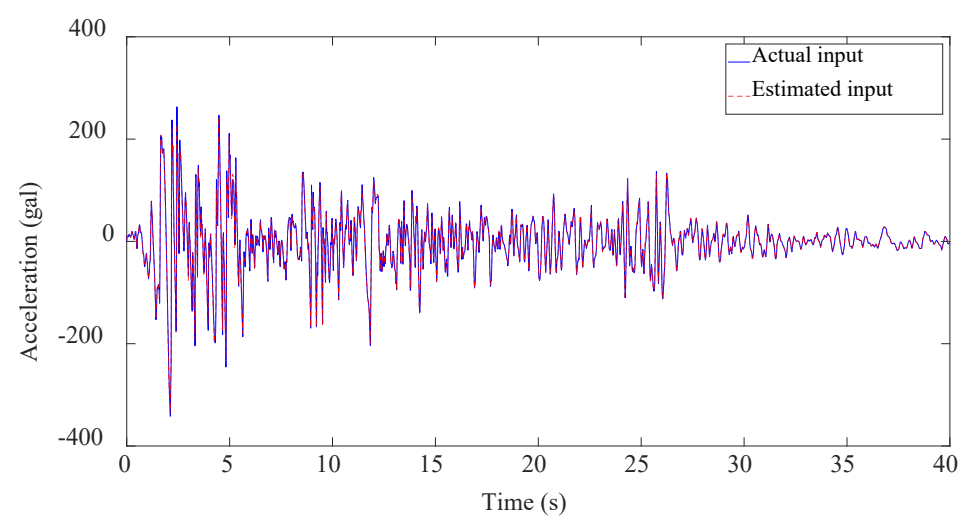

(a) Estimated input

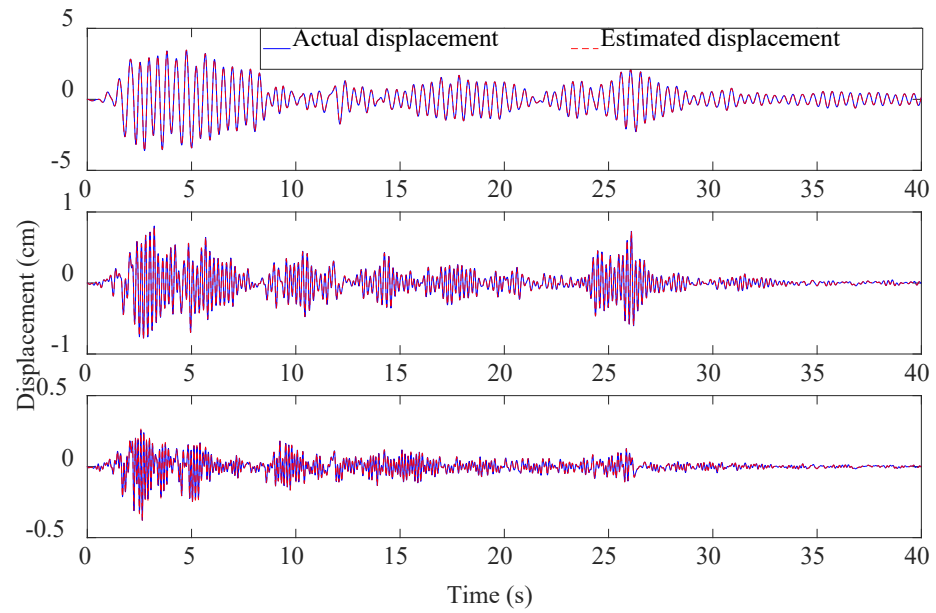

(b) Estimated modal displacement responses of first (top), second (middle), and third (bottom) modes
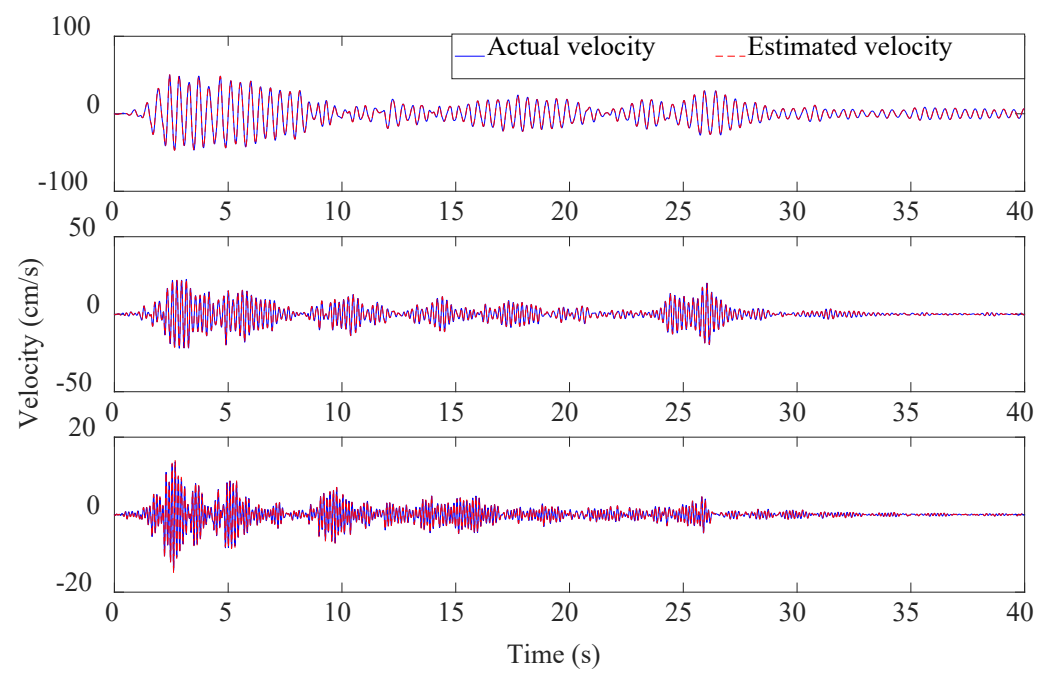

(c) Estimated modal velocity responses

of first (top), second (middle), and third (bottom) modes

Figure 3. Input and state estimation using modified Kalman filter 
Next, the relative displacements of all floors were calculated using Equation (10). As shown in Figure 4, the estimated time history responses match the actual responses, indicating a good estimation of structural deformation. To consider the effect of environmental noise and investigate the robustness of the proposed approach, the responses were calculated at the $5 \%$ and $10 \%$ levels of the signal RMS noise. The maximum relative displacement was extracted, and subsequently the maximum inter-story drift was determined. As can be seen in Figure 5, the algorithm performs well at noise level of $5 \%$, and the efficiency will decline as the noise increases. In addition, the estimation of relative displacements of all floors and the inter-story drifts of the bottom floors is robust against the disturbances, and the estimation of inter-story drift of the floor with minor deformation is susceptible to noise.

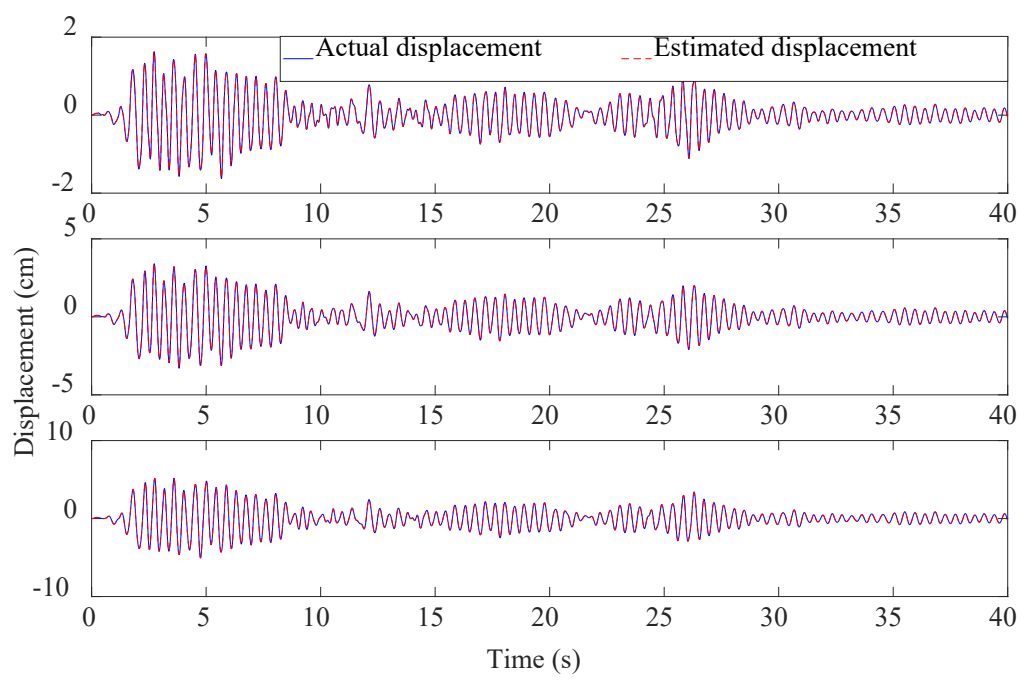

Figure 4. Estimated displacements of first (top), second (middle), and third (bottom) floors

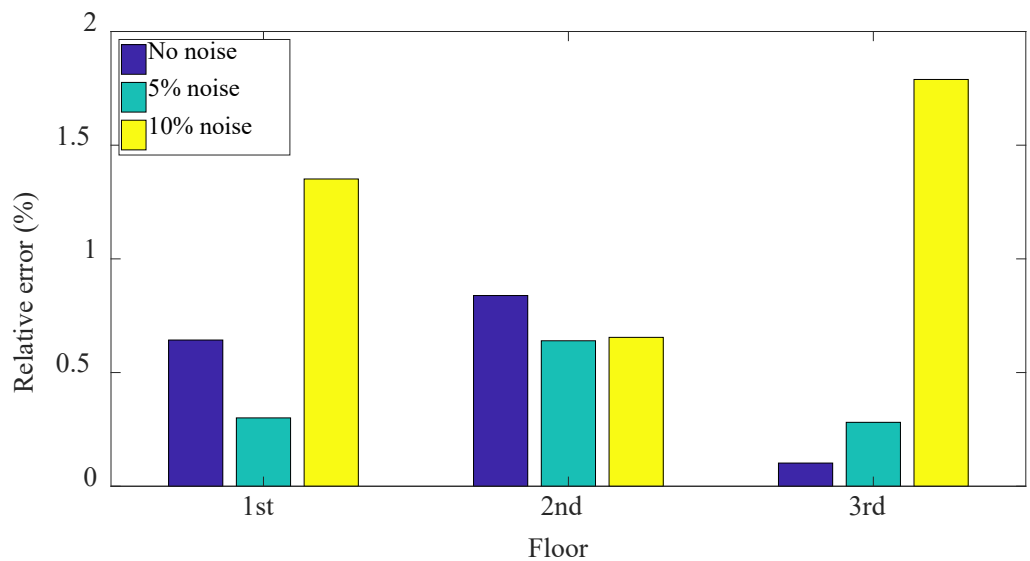

(a) Estimated relative displacement 


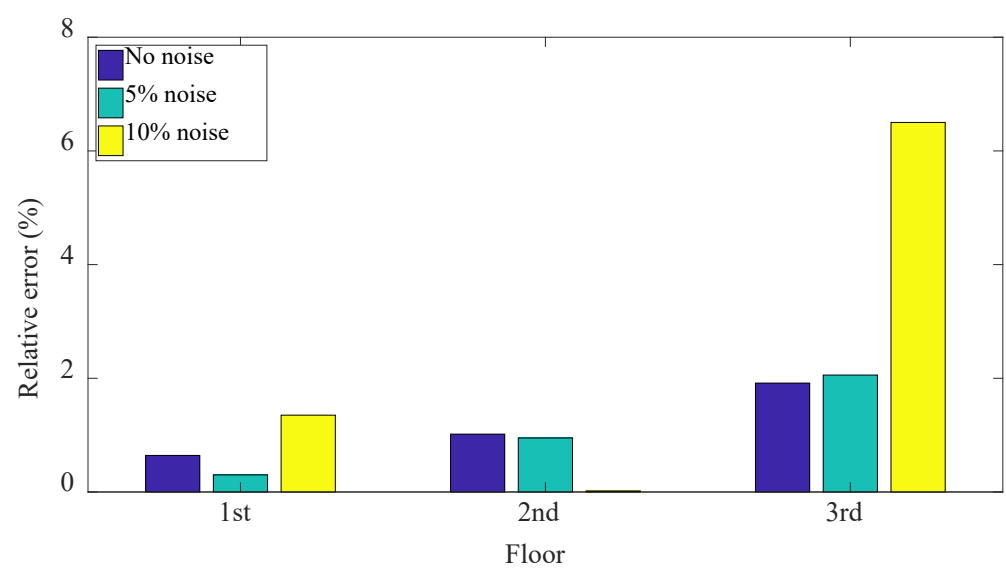

(b) Estimated inter-story drift

Figure 5. Relative maximum error for different noise levels

\section{Conclusion}

This paper presented a way to estimate the relative displacement time history and maximum inter-story drift of MDOF shear structures merely from the absolute acceleration of a single floor recorded by a sensor. Numerical simulations proved that the system state and undetected input can be simultaneously and accurately estimated based on the state-space system, and the relative displacement time history and maximum inter-story drift can also be obtained with a small error even under strong environmental disturbances, providing reliable evidence for making structural assessments.

On the other hand, in the paper we just investigate a simple scenario in which the modal parameters are known, the structure is linear and mildly damped. In the future, we will focus on the estimation of maximum inter-story drift of the seismic isolation structures and supplementally damped buildings with the unknown modal information.

\section{Acknowledgements}

The study was partially supported by the 'Design the Future' Award of Keio University and the KLL (Keio Leading-edge Laboratory) 2019 Ph.D. Program Research Grant.

\section{References}

[1] Mita A 2003 Structural dynamics for health monitoring Nagoya, Japan: Sankeisha

[2] 2010 Code for seismic design of building. China: Ministry of Housing and Urban-Rural Development of the People's Republic of China

[3] 2013 The building standard law. Japan: The Building Center of Japan

[4] 2010 Minimum design loads for buildings and other structures. USA: American Society of Civil Engineers

[5] Gomez F, Park J W, Spencer Jr B F 2018 Reference - free structural dynamic displacement estimation method. Structural Control and Health Monitoring, 25 e2209.

https://doi.org/10.1002/stc.2209 
[6] Kasai K, Mita A, Kitamura H, et al 2013 Performance of seismic protection technologies during the 2011 Tohoku-Oki earthquake. Earthquake Spectra, 29 S265-S293. https://doi.org/10.1193/1.4000131

[7] Dan M, Ishizawa Y, Tanaka S, et al 2015 Vibration characteristics change of a base-isolated building with semi-active dampers before, during, and after the 2011 Great East Japan earthquake. Earthquake and Structures, 8 889-913. https://doi.org/10.12989/eas.2015.8.4.889

[8] Siringoringo D M, Fujino Y 2015 Seismic response analyses of an asymmetric base isolated building during the 2011 Great East Japan (Tohoku) Earthquake. Structural control and health monitoring, 22 71-90. https://doi.org/10.1002/stc.1661

[9] Tong Y, Xie L, Xue S, et al 2017 Performance of a passively-controlled steel building before and after the 2011 great east japan earthquake. Proceedings of the Eleventh International Workshop on Structural Health Monitoring (California, USA. https://doi.org/10.12783/shm2017/14134)

[10]Brewick P T, Johnson E A, Sato E, et al 2018 Constructing and evaluating generalized models for a base - isolated structure. Structural Control and Health Monitoring, 25 e2243. https://doi.org/10.1002/stc.2243

[11]Feng D, Feng M Q 2016 Vision - based multipoint displacement measurement for structural health monitoring. Structural Control and Health Monitoring, 23 876-890. https://doi.org/10.1002/stc.1819

[12] Gillijns S, De Moor B 2007 Unbiased minimum-variance input and state estimation for linear discrete-time systems with direct feedthrough. Automatica, 43 934-937. https://doi.org/10.1016/j.automatica.2006.11.016

[13]Azam S E, Chatzi E, Papadimitriou C 2015 A dual Kalman filter approach for state estimation via output-only acceleration measurements. Mechanical Systems and Signal Processing, 60 866-886. https://doi.org/10.1016/j.ymssp.2015.02.001

[14]Xue S, Xu K, Liyu X, et al 2019 Crack sensor based on patch antenna fed by capacitive microstrip lines. Smart Materials and Structures, 28 85012. https://doi.org/10.1088/1361$665 \mathrm{X} / \mathrm{ab} 2834$

[15] Tchafa F M, Huang H 2018 Microstrip patch antenna for simultaneous strain and temperature sensing. Smart Materials and Structures, 27 65019. https://doi.org/10.1088/1361$665 \mathrm{X} / \mathrm{aabd} 47$

[16] Yoshimoto R, Mita A, Okada K 2005 Damage detection of base - isolated buildings using multi - input multi - output subspace identification. Earthquake engineering \& structural dynamics, 34 307-324. https://doi.org/10.1002/eqe.435

[17]Clough R W, Penzien J 2005 Dynamics of structures USA: Computers \& Structures, Inc 